Meta

Journal des traducteurs

Translators' Journal

\title{
Sur la notion de contexte
}

\section{Gaston Gross}

Volume 55, numéro 1, mars 2010

Le parcours du sens : d'une langue à l'autre — Mélanges offerts à André Clas

The Way of Meaning: From a Language to Another - Collection of Articles Offered to André Clas

URI : https://id.erudit.org/iderudit/039612ar

DOI : https://doi.org/10.7202/039612ar

Aller au sommaire du numéro

\section{Éditeur(s)}

Les Presses de l'Université de Montréal

ISSN

0026-0452 (imprimé)

1492-1421 (numérique)

Découvrir la revue

Citer cet article

Gross, G. (2010). Sur la notion de contexte. Meta, 55(1), 187-197.

https://doi.org/10.7202/039612ar

\section{Résumé de l'article}

Le présent article constitue une réflexion sur la notion de contexte, qui est l'une des plus importantes de l'analyse linguistique, dans la mesure où presque tous les prédicats sont polysémiques et que seule la distribution permet de déterminer la lecture adéquate. Mais le contexte ne peut pas être réduit à l'environnement immédiat de gauche et de droite de l'élément à analyser : seule la détermination des relations argumentales permet de définir un opérateur avec précision. Or, un texte n'est pas constitué d'une suite de phrases simples dans leur ordre canonique. Aussi est-il nécessaire d'éliminer les contextes immédiats qui ne participent à cette reconnaissance. Il peut s'agir d'incises, de structures figées, etc. Il faut donc être en mesure de trouver les relations prédicat-arguments qui caractérisent les phrases simples. Cette recherche repose sur l'utilisation de dictionnaires électroniques qui comprennent ces informations. Ces dictionnaires sont fondés sur les classes d'objets. Celles-ci permettent, entre autres, de montrer que dans la reconnaissance des emplois les compléments sont plus discriminants que les sujets. La détection de la relation prédicat-arguments est encore facilitée si on a recensé, pour chaque emploi, toutes les restructurations qu'un schéma d'arguments est en mesure de subir. La notion de contexte ne constitue donc pas une délimitation mécanique que l'on peut effectuer en sélectionnant un nombre déterminé de mots à gauche et à droite mais relève d'une analyse qui met en jeu la totalité des informations syntaxiques.
Ce document est protégé par la loi sur le droit d'auteur. L'utilisation des services d'Érudit (y compris la reproduction) est assujettie à sa politique d'utilisation que vous pouvez consulter en ligne.

https://apropos.erudit.org/fr/usagers/politique-dutilisation/ 


\title{
Sur la notion de contexte
}

\author{
GASTON GROSS \\ LDI (UMR 7187, CNRS) - Université Paris 13, Paris, France \\ gaston.gross@wanadoo.fr
}

\section{RÉSUMÉ}

Le présent article constitue une réflexion sur la notion de contexte, qui est l'une des plus importantes de l'analyse linguistique, dans la mesure où presque tous les prédicats sont polysémiques et que seule la distribution permet de déterminer la lecture adéquate. Mais le contexte ne peut pas être réduit à l'environnement immédiat de gauche et de droite de l'élément à analyser: seule la détermination des relations argumentales permet de définir un opérateur avec précision. Or, un texte n'est pas constitué d'une suite de phrases simples dans leur ordre canonique. Aussi est-il nécessaire d'éliminer les contextes immédiats qui ne participent à cette reconnaissance. II peut s'agir d'incises, de structures figées, etc. II faut donc être en mesure de trouver les relations prédicat-arguments qui caractérisent les phrases simples. Cette recherche repose sur l'utilisation de dictionnaires électroniques qui comprennent ces informations. Ces dictionnaires sont fondés sur les classes d'objets. Celles-ci permettent, entre autres, de montrer que dans la reconnaissance des emplois les compléments sont plus discriminants que les sujets. La détection de la relation prédicat-arguments est encore facilitée si on a recensé, pour chaque emploi, toutes les restructurations qu'un schéma d'arguments est en mesure de subir. La notion de contexte ne constitue donc pas une délimitation mécanique que l'on peut effectuer en sélectionnant un nombre déterminé de mots à gauche et à droite mais relève d'une analyse qui met en jeu la totalité des informations syntaxiques.

\begin{abstract}
This paper addresses the notion of context, which is one of the most important concepts in linguistic analysis inasmuch as all predicates are polysemous, which entails that only their distribution can determine the appropriate interpretation. But context cannot be reduced simply to the words occurring to the left and to the right of the ambiguous element in question: only the determination of the argumental relations are sufficient to define operators with precision. Moreover, a text is not in general simply a sequence of elementary sentences in their canonical order. It is therefore necessary to eliminate those parts of the context that do not contribute directly to the identification of the right interpretation. These include insertions, frozen constructions, etc. One must be able to identify the predicate argument structures that underlie the elementary sentences. This task is based on the use of electronic dictionaries which contain the relevant information. These dictionaries are constructed on the basis of object classes. Among other things, these classes also show that to identify interpretations of predicates, complements are more discriminative than subjects. The recognition of predicate argument structures can be enhanced by an inventory, relative to each interpretation, of the restructurings that each argument schema can undergo. The notion of context should therefore not be regarded as a mechanical demarcation that can be carried out by selecting a certain number of words to the left and the right, but rather as an analytic procedure which involves the totality of syntactic information available.
\end{abstract}

\section{MOTS-CLÉS/KEYWORDS}

contexte, emploi, relation prédicat-arguments, classes d'objets, restructuration context, usage, predicate argument structure, object classes, restructuring 


\section{1. À propos de définition}

Un exercice scolaire classique consiste à demander à un élève de définir le sens d'un mot. Cet exercice peut sembler banal, mais il pose des questions de fond de nature théorique. Imaginons que la question porte sur le sens du verbe casser. Il est impossible de répondre de façon satisfaisante à cette question. Admettons qu'un élève réponde briser. On comprend que cet élève a sélectionné, de façon arbitraire, un des sens du verbe casser et qu'il en a proposé un synonyme. Il est bien connu cependant que la plupart des verbes sont polysémiques, comme d'ailleurs la plupart des lexèmes. Il ne serait donc pas étonnant qu'un autre élève propose fracturer ou un autre encore annuler. Chacun d'eux aura sélectionné parmi les différents sens du verbe casser celui qui lui est connu ou qui lui vient le plus facilement à l'esprit au moment de la question. Le maître ne devrait pas se contenter de ces réponses. Il devrait demander à chacun les conditions dans lesquelles le verbe casser prend telle ou telle signification et de fournir une phrase qui la met en lumière. On verrait ainsi que casser signifie briser avec des compléments comme verre, vaisselle; fracturer avec un complément désignant un $<$ membre $>$ comme jambe ou bras; et annuler si l'objet fait partie de la classe des $<$ contrats $>$ ou des < actes juridiques $>$. Comme le verbe casser est polysémique, il aurait fallu demander: "Quels sont les différents sens du verbe casser? Donnez un synonyme pour chacun de ces sens». Hors contexte donc, il est impossible de reconnaître la signification d'un mot. Comment donc définir le sens d'un verbe ou de tout autre élément lexical ? La réponse est bien connue: c'est le contexte qui détermine le sens d'un terme (Gross 1994a). Ce qui vient d'être dit pour un mot de la langue générale (casser) vaut tout autant pour un lexème d'une langue de spécialité, c'est-àdire pour un terme. Il est donc acquis que pour comprendre le sens d'un mot, il faut prendre en considération son environnement.

\section{Conséquences théoriques}

Les constatations que l'on peut faire à partir de cet exercice sont les suivantes:

- Le lexique ne peut pas être séparé de la syntaxe, c'est-à-dire de la combinatoire des mots (Gross 1975);

- La sémantique n'est pas autonome non plus: elle est le résultat de la combinaison des éléments lexicaux organisés d'une certaine façon (distribution, Gross 1981);

- On ne peut postuler sérieusement qu'il existe trois niveaux indépendants dans la description linguistique, celui du lexique, de la syntaxe et de la sémantique, car on ne voit pas comment ils pourraient être articulés, s'ils étaient indépendants;

- L'existence de la polysémie, qui est une des propriétés fondamentales des langues naturelles, oblige à relier ces trois niveaux, ce qui ne peut se faire que sur la base du lexique. Cet amalgame s'opère dans la notion d'emploi, qui est un des concepts les plus importants de la grammaire;

- Un emploi, représenté par une phrase simple, est constitué par un prédicat avec un schéma d'arguments déterminé. Cet ensemble a des propriétés spécifiques, différentes de celles que pourrait avoir cette forme prédicative dans un autre environnement;

- La description d'un emploi implique la reconnaissance de son contexte, c'est-à-dire de son environnement approprié, autrement dit de sa distribution. 


\section{Contextes aléatoires}

Comme aucun prédicat ne peut être défini hors de son contexte, la question se pose de savoir comment déterminer ce contexte. On peut être tenté de dire que toutes les informations nécessaires à la description d'un mot sont définies par l'ensemble de ses environnements que l'on peut trouver dans de grands corpus et qu'il suffit d'avoir recours à eux pour savoir comment ces environnements nous permettent de sélectionner le sens d'un mot parmi tous les autres sens possibles. Cette recherche ne peut pourtant pas être mécanique. Il ne suffit pas de donner comme requête à un logiciel de sélectionner les cinquante caractères qui précèdent ou qui suivent un verbe, par exemple, pour connaître sa construction. En effet, les éléments qui définissent un mot ne sont pas nécessairement contigus, comme on le postule par définition pour les éléments de la phrase simple, les arguments définissant l'emploi d'un prédicat.

Il y a souvent entre un prédicat et ses arguments des insertions de diverses natures qui n'appartiennent pas à la structure pertinente et qu'il faut ignorer si l'on veut retrouver la distribution qui éclaire le terme en question. Ces insertions peuvent être:

a) des incises (Fairon 2000):

- Il faut tailler, comme on le sait, les arbres au début du printemps.

- Louis XIV, à ce qu'on dit, a réduit le pouvoir des nobles.

- Il serait, à ce que j'ai cru comprendre, dans un triste état.

- L'État, nous le savons tous, est un monstre froid.

b) des négations:

- Les adolescents ne jouent plus aux billes.

- Il n'a répondu, sur aucun point, à mes questions.

c) des adverbes:

- Pierre a participé grandement à cette victoire.

- Paul a changé radicalement de conduite.

d) des circonstancielles:

- Pierre a oublié, avant de partir, de fermer le gaz.

e) des déterminants nominaux:

- Paul a lu un tas de livres.

- Il y avait à l'arrière du front un monceau de cadavres.

- Il a versé dans son thé un nuage de lait.

Dans ces phrases, le système doit pouvoir:

- reconnaître que l'environnement de droite de tailler n'est pas la suite comme on le sait mais le substantif arbres;

- discerner que l'environnement de droite de Louis XIV est a réduit et que à ce qu'on dit n'est pas pertinent pour la description du verbe réduire;

- constater que la négation dans b) est une règle générale de la grammaire et qu'elle ne joue aucun rôle dans la définition syntaxique de jouer, bien qu'il puisse arriver qu'une négation fasse partie de la structure même de la phrase simple comme dans: n'en faire qu'à sa tête; ne pas en rater une (Gross 1998);

- savoir que les adverbes grandement, radicalement ne font pas partie du schéma d'arguments des prédicats participer ou changer, contrairement, par exemple, aux 
constructions suivantes, où l'adverbe est obligatoire et constitue pour ainsi dire un argument: se comporter Adv, présenter bien, bien/mal accueillir que;

- être capable de reconnaître les propositions et compléments circonstanciels qui s'insèrent à l'intérieur d'un schéma d'arguments: avant de partir doit être mis entre virgules pour pouvoir relier oublié et son complément fermer le gaz;

- être en mesure de considérer un tas de et un monceau de comme des déterminants nominaux quantifieurs et non des compléments du verbe lire, par exemple (Buvet 1998).

On voit que si on définit les prédicats par la nature de leurs arguments, on est obligé de ne pas tenir compte de l'environnement immédiat et d'aller chercher la suite représentant effectivement le complément réel. La distribution d'un mot n'est pas constituée par son contexte matériellement immédiat mais doit faire l'objet d'une analyse permettant de trouver les termes qui font cohésion.

\section{Contextes contraints}

Il y a des cas où l'environnement immédiat, calculé en termes de catégories grammaticales, n'est pas non plus éclairant pour la détermination de la construction d'un prédicat. C'est le cas des constructions figées (Gross 1993).

\subsection{Insertions dans les suites figées}

Maurice Gross (1975) a signalé que les constructions verbales figées n’ont pas une structure interne différente de celle des constructions régulières, en termes de séquences catégorielles. Elles acceptent donc souvent des insertions du type qu'on vient de voir:

- Pierre prend toujours des vessies pour des lanternes.

- La moutarde lui a, semble-t-il, monté au nez.

Voilà une première difficulté qu'il faut pouvoir résoudre. La possibilité d'insertion n'est donc pas un critère sûr de compositionnalité.

\subsection{Un environnement contraint et non compositionnel}

Indépendamment de ce fait, dans les constructions figées, le contexte de gauche ou de droite peut constituer un faux environnement, puisque le sens du prédicat n'est pas déterminé par la nature sémantique des substantifs qui y figurent. C'est l'ensemble constitué par le verbe et la séquence des substantifs qui le suivent qui forme une unité sémantique, de sorte qu'on ne peut pas analyser le substantif à la droite du verbe comme un de ses arguments. On ne peut pas considérer pipe comme un vrai complément de casser dans casser sa pipe. Le problème de l'environnement se pose alors non pas à l'intérieur de la suite figée mais au niveau externe, puisque celle-ci joue le rôle d'une catégorie grammaticale complexe. Ce verbe complexe peut, à son tour, avoir de vrais arguments (Gross 1996b). Les trois verbes figés suivants ont tous un complément humain:

- (Tirer les vers du nez) à Nhum

- (Donner du fil à retordre) à Nhum

- (Apporter de l'eau au moulin) de Nhum 


\section{Contextes significatifs}

La détermination du sens d'un prédicat à l'aide du contexte n'est donc pas une opération simple. Le contexte n'est jamais une donnée immédiate quand il s'agit de textes, qui ne sont jamais des suites de phrases simples, telles qu'elles sont définies dans les grammaires: des prédicats accompagnés de la suite la plus longue de leurs arguments. Et pourtant la phrase simple est l'unité la plus importante des textes. Le premier travail d'analyse consiste donc à reconnaître toutes les phrases simples d'un texte. C'est par leur reconnaissance que l'on peut mettre au point les environnements adéquats. Cette opération ne peut se faire que sur la base d'un dictionnaire électronique des prédicats qui doit comprendre pour chacun d'eux les informations suivantes (Gross 1994a):

- sa classe sémantique;

- ses arguments: leur nombre, leur nature sémantique et leur possible effacement;

- sa forme morphologique et les passages possibles de l'une à l'autre (ou aux autres) de ces formes (aimer, amour, amoureux);

- la forme de son actualisation (conjugaison, verbe support pour les prédicats nominaux);

- la nature de son aspect interne (mode d'action);

- la détermination acceptée par les arguments, en fonction de leur classe sémantique;

- la compatibilité aspectuelle entre le prédicat et ses arguments.

Toutes ces informations doivent figurer dans un dictionnaire électronique. L'analyse d'un texte consiste alors à reconnaître les structures phrastiques qui figurent dans ce texte à partir de la description exhaustive des prédicats répertoriés dans le dictionnaire électronique.

\section{Les classes d'objets comme outils de discrimination du sens des prédicats}

Les différents emplois d'un verbe et, de façon générale, de tout prédicat peuvent être mis en évidence à l'aide des classes d'objets. Une classe d'objets est un ensemble de substantifs, sémantiquement homogènes, qui déterminent une rupture d'interprétation d'un prédicat donné, en délimitant un emploi spécifique. Selon cette définition, les classes d'objets ne sont donc pas des concepts sémantiques abstraits mais des entités construites sur des bases syntaxiques et déterminées par la signification des prédicats. Nous donnons ci-dessous, pour deux verbes et deux adjectifs, les schémas distributionnels des différents emplois, caractérisés en classes d'objets pour les sujets (NO) et pour les compléments (N1).

\section{Verbes:}

Allonger / N0: <couturier $>$ / N1: <vêtements $>$ / N2: de $<$ hum $>$

Allonger / N0 : <cuisinier $>$ / N1: < potages $><$ sauces $>$ / N2: avec $<$ huile $><$ bouillon $>$ <eau>

Allonger / N0: <hum $>/ \mathrm{N} 1:<$ argent $><$ pourboire $>/ \mathrm{N} 2:$ à $<$ hum $>$

Allonger / N0: <hum $>/ \mathrm{N} 1:<$ hum $>/ \mathrm{N} 2$ : sur $<$ sol $><$ lit $><$ civières $>$

Atteindre / N0: $<$ hum $>/ \mathrm{N} 1:<$ loc $>$

Atteindre / N0: <hum $>/ \mathrm{N} 1:<$ âge $>$ 
Atteindre / N0: <hum $>$ / N1: <but $>$

Atteindre / N0: <eau $>/ \mathrm{N} 1:<$ niveau $>$

Atteindre / N0: <hum $>$ / N1: <hum $>$ / N2: à <téléphone $>$

Atteindre / N0: <projectile $>$ / N1: $<$ hum $><$ cible $>$

Atteindre / N0: $<$ maladie $>$ / N1: $<$ hum $>$

Atteindre / N0: $<$ hum $>/ \mathrm{N} 1:<$ public $>$

Atteindre / N0: <malheur $>$ N1: $<$ hum $>$

Adjectifs:

Juste / N0: <hum > / Sy: droit, intègre, honnête

Juste / N0: <hum> / N1: avec <hum > / Sy: équitable, impartial

Juste / N0: <action > Sy: équitable

Juste / N0: <récompense, punition> / Sy: légitime, justifié, fondé

Juste / N0: <calcul, déduction> / Sy: correct, exact

Juste / N0: <vêtement > / Sy: étroit, étriqué

Juste / N0: <instr de mesure > Sy: exact, précis

Juste / N0: <instr de musique> / Sy: accordé

Âpre / N0: <objet concret> / Sy: rugueux

Âpre / N0: <lieu géologique> / Sy: escarpé, raboteux

Âpre / N0: <fruit> / Sy: acide, âcre

Âpre / N0: <dispute> / Sy: violent, rude

Âpre / N0: <propos> / Sy: véhément

Âpre / N0: <combat> / Sy: violent, sanglant, acharné, sauvage

Âpre / N0: <climat> / Sy: rude, rigoureux

\section{Disparité entre les positions argumentales. Les compléments sont plus importants que les sujets}

Comment mettre en évidence les différents sens d'un verbe comme casser? On a coutume de dire que c'est la nature des arguments qui permet de discriminer les différents polysèmes. C'est mettre sur le même plan le sujet et les compléments.

\subsection{Information faible apportée par les sujets}

Or, les compléments sont beaucoup plus importants que les sujets pour déterminer le sens d'un prédicat, c'est-à-dire pour déterminer son emploi. Si je dis que le verbe casser, qui a plus d'une dizaine d'emplois, possède un sujet humain, je peux discriminer celui des emplois qui a un sujet événementiel: Ce contretemps a cassé le rythme, mais cette information ne permet pas de séparer entre eux les autres emplois dont le sujet est humain. On voit à travers cet exemple que la valeur informative du sujet n'est pas très grande.

\subsection{Information riche apportée par les compléments}

Examinons maintenant les compléments. Si le complément est un nom de la classe des <objets cassables>: L'enfant a cassé la bouteille, on sait immédiatement qu'il s'agit de /briser un objet fragile/. Si le complément est un élément de la classe des $<$ membres du corps humain $>$, alors le verbe signifie /fracturer/. Si le complément est un humain, le verbe signifie /briser l'espoir de qq/. Si le complément désigne un officier ou représente un nom de fonction, alors le verbe casser peut être paraphrasé par dégrader. 
Enfin, si le complément est un élément de la classe des <chaussures>, alors le verbe signifie /assouplir/.

Cette analyse n'est pas propre au verbe casser. Prenons un dernier exemple. Il est presque impossible de préciser ce que veut dire: Paul arrange... L'intuition ne nous aide pas beaucoup. L'indication de la nature sémantique du sujet a donc là encore un faible indice de discrimination. Observons maintenant la valeur discriminatoire des compléments: arranger des papiers, arranger un morceau de musique, arranger un appartement, arranger des fleurs dans un vase, arranger un voyage, arranger un conflit. Le sens est clair à chaque fois. Il existe une ambiguïté quand l'objet est un humain arranger Paul. Les deux interprétations dépendent alors de la nature du sujet: On l'a salement arrangé, Cela arrange Paul. La démonstration serait encore plus éclairante avec le verbe prendre. Le sujet ne permet de reconnaître l'emploi que dans peu de cas: Cela prend $=($ Le béton, la mayonnaise $)$ prend. Avec l'indication du seul sujet humain, ce verbe ne peut pas être interprété, ce qui est le cas, en revanche, avec le complément: prendre un aliment, prendre un moyen de transport individuel, prendre un moyen de transport en commun, prendre une voie, prendre une place forte, prendre un objet, sans compter les emplois comme support: prendre plaisir à, prendre une cuite, etc.

La conclusion à tirer, c'est que l'environnement de droite, c'est-à-dire les compléments, apporte beaucoup plus d'informations que le sujet dans l'interprétation d'un prédicat. Il ne s'agit pas seulement de significations différentes, en fonction de la classe sémantique des compléments, c'est le prédicat lui-même qui a des propriétés spécifiques en fonction de cette classe: c'est ce que nous appelons un emploi. Cette observation d'importance vaut évidemment aussi pour les langues de spécialités.

\section{La notion de prédicats appropriés}

Nous avons vu que la nature sémantique des arguments permet de rendre compte du sens d'un prédicat dans un contexte. On peut procéder de la même façon pour la description des substantifs eux-mêmes. Soit le mot chaîne. Ce mot a, dans le Petit Robert $\left(1993^{1}\right)$, une dizaine de sens différents. Ces différentes significations peuvent être accompagnées de verbes qui ne les discriminent pas, comme:

- des verbes de perception: voir, apercevoir;

- ou des verbes généraux comme: parler de, apprécier, aimer: J'aime beaucoup cette chaîne.

Mais il existe des verbes, appelés verbes appropriés, qui permettent de déterminer le sens d'un substantif parmi les autres possibles. Ainsi le sens peut-être le plus fréquent du mot chaîne est illustré par un exemple comme: J'ai attaché mon vélo à la grille avec une chaîne. Certains types de chaînes servent à attacher ou fixer des objets spécifiques. Il s'agit soit de certains animaux: promener son chien à la chaîne (laisse) soit de certains types d'humains: On avait mis les bagnards à la chaîne. Un autre emploi de chaîne est illustré par le verbe actionner: Cette roue est actionnée par une chaîne. Dans ce cas, le mot désigne un moyen de transmission. Porter autour $d u$ (cou, bras): implique que le substantif est synonyme de collier ou gourmette. On pourra répliquer qu'on peut très bien avoir une chaîne de vélo autour de son cou, mais alors on n'emploiera pas le verbe porter mais mettre.

Un autre emploi est illustré par des verbes comme: écouter de la musique sur cette chaîne (chaîne haute-fidélité), allumer/éteindre une chaîne. En général, un seul 
prédicat ne suffit pas à définir le sens d'un substantif. Une classe de substantifs est donc définie par une grappe de prédicats qui lui sont strictement appropriés.

\section{Contextes potentiels}

Les dictionnaires électroniques décrivent les éléments lexicaux à l'aide d'un certain nombre de propriétés qui figurent dans les différents champs d'une base de données. Ces informations sont, comme nous venons de le voir, des contextes strictement appropriés délimitant un emploi, par rapport à tous les autres emplois également possibles. Mais les propriétés des mots ou termes ne sont pas entièrement prises en charge par les informations distributionnelles que nous avons présentées dans ce qui précède et qui figurent dans les dictionnaires électroniques. D’autres propriétés permettent de caractériser les emplois, elles sont de nature transformationnelle.

\subsection{Propriétés non prédictibles}

Parmi ces autres propriétés, certaines sont prédictibles à partir de la classe sémantique du prédicat, d'autres non.

\subsubsection{Forme morphologique des prédicats}

Parmi celles qui ne peuvent pas être prédites figure le fait pour un prédicat d'avoir plusieurs formes morphologiques: verbe, nom, adjectif. Si l'on connaît la classe sémantique d'un prédicat, on n'est pas en mesure de connaître les formes morphologiques qu'il peut revêtir. Une des classes qui a la plus grande régularité de ce point de vue est celle des <sentiments $>$. Un grand nombre de prédicats de ce type ont les trois formes aimer, amour, amoureux; respecter, respect, respectueux. Mais d'autres n'ont que deux formes, honte, honteux. Et d'autres n'ont qu'une seule forme: il n'y a pas de forme verbale à ressentiment ou pitié. Il existe aussi de faux parallélismes: aviser n'est pas l'équivalent de avis ni parole de parler. Ces indications doivent figurer évidemment dans un dictionnaire électronique.

\subsubsection{Rotation des arguments}

Une autre propriété non prédictible est ce que l'on pourrait appeler la rotation des arguments (Gross 1975). On ne parle pas ici d'une restructuration qui a été appelée permutation de longueur et qui est illustrée ci-après. L'ordre canonique veut qu'un complément direct précède un complément indirect:

- J’ai apporté ce cahier à Paul.

- ?J'ai apporté à Paul ce cahier.

Mais quand le complément direct est très long, alors on inverse l'ordre: J'ai apporté à Paul le cahier que j'ai promis lors de notre dernière rencontre. En revanche, il existe d'autres déplacements qui semblent appartenir en propre à certains prédicats et qui ne sont donc pas prédictibles. C'est le cas du verbe livrer:

- Paul a livré du charbon à Jean.

- Paul a livré Jean en charbon. 
Dans ce cas, le verbe reste le même. Mais il existe des couples de verbes qui permettent de mettre l'accent sur tel complément ou tel autre. Il s'agit souvent de verbes dits datifs mais ce n'est pas le cas de tous. Ainsi, on peut faire correspondre:

- La nature a donné à Paul une bonne santé.

- La nature a doté Paul d'une bonne santé.

- Paul a dit à Jean qu'il y a un délai de trois jours.

- Paul a informé Jean qu'il y a un délai de trois jours.

\subsubsection{Effacement des arguments}

Dans un dictionnaire électronique, on note soigneusement la suite la plus longue des arguments d'un prédicat. Mais on sait que, dans les textes, cette suite ne figure pas toujours intégralement. Cet effacement dépend du prédicat. Il est clair qu'un verbe comme manger peut figurer sans complément: Paul est en train de manger. Mais ce n'est pas le cas du verbe prendre: Paul a pris un steak: ${ }^{\star}$ Paul a pris, sauf dans l'emploi technique d'un jeu de cartes: Je prends, ou encore quand le sujet représente des mots comme: (La mayonnaise, le béton) a pris. Le problème mérite attention, car dans certains cas, l'effacement d'un argument change le sens du prédicat: Ce chiffon sent (bon, mauvais); ce chiffon sent = sent mauvais. Bien que la possibilité d'une mise au passif soit beaucoup plus régulière, il faut tout de même le noter dans le dictionnaire, puisqu'un assez grand nombre de verbes transitifs directs n'ont pas de forme passive: Ce chiffon sent la lavande, ${ }^{\star}$ La lavande est sentie par ce chiffon.

\subsubsection{Constructions croisées}

Les constructions croisées impliquent une thématisation d'un complément $<$ locatif $>$. La table 34 LO du LADL (Boons, Guillet et al. 1976) recense des constructions mettant en jeu des verbes dont les arguments peuvent être inversés:

- Les abeilles grouillent dans le jardin.

- Le jardin grouille d'abeilles.

Cette restructuration n'affecte pas tous les verbes locatifs:

- Les outils traînent dans l'atelier.

- ${ }^{*}$ L'atelier traîne d'outils.

\subsubsection{Question cachée}

D’autres propriétés potentielles sont à décrire, comme la question cachée. Les emplois qui séparent les verbes savoir et connaître ont été souvent étudiés (voir Gross 1996b). Il existe des environnements où les deux verbes semblent avoir le même emploi : Paul sait la solution, Paul connaît la solution. Pourtant, savoir a une propriété que n'a pas connaître. Cette propriété a été appelée la question cachée: Paul sait quelle est la solution, ${ }^{*}$ Paul connaît quelle est la solution.

\subsection{Propriétés prédictibles}

Nous examinons maintenant des propriétés qui sont le fait de classes sémantiques, donc prédictibles, et qu'il n'est pas indispensable de noter dans un dictionnaire pour chaque élément lexical concerné. Comme le phénomène touche l'ensemble des éléments 
d'une classe donnée, elles peuvent figurer dans la grammaire et ne sont notées de ce fait qu'une seule fois. Elles n'en sont pas moins des propriétés dont il faut disposer si l'on veut reconnaître toutes les phrases d'un texte. Nous donnons ici quelques exemples.

\subsubsection{Les verbes causatifs de sentiments}

Maurice Gross (1975) a montré dans la table 4 de cet ouvrage que ces verbes ont la possibilité d'avoir une construction disloquée. Ils ont, dans la construction canonique, un sujet phrastique, qui devient un complément prépositionnel en par:

- Le sang-froid (que Paul a, de Paul) a surpris Jean.

- Paul a surpris Jean par son sang-froid.

\subsubsection{La classe des verbes symétriques (qui est caractérisée par des} restructurations spécifiques)

Soit le verbe se marier, où l'on observe les constructions suivantes:

- Paul se marie avec Jeanne.

- Jeanne se marie avec Paul.

- Paul et Jeanne se marient (l'un avec l'autre, ensemble).

- Jeanne et Paul se marient (l'un avec l'autre, ensemble).

- Paul et Jeanne se marient.

La symétrie peut aussi concerner les compléments:

- Paul compare la copie avec l'original.

- Paul compare l'original avec la copie.

- Paul compare l'original et la copie.

\subsubsection{Thématisation de l'objet}

On peut rapprocher de cela les constructions converses, qui transforment un complément datif en sujet:

- Paul a vendu une voiture à René.

- René a acheté une voiture à Paul.

- Paul a donné un cahier à René.

- René a reçu un cahier de Paul.

- Paul a donné une raclée à René.

- René a reçu une raclée de Paul.

\section{Conclusion}

Les conclusions qu'on peut tirer de ces analyses sont multiples. Tout d'abord, le fait que les niveaux d'analyse généralement postulés dans la description grammaticale soient en fait totalement interdépendants a pour conséquence que l'unité minimale d'analyse n'est pas le mot, mais la phrase simple. Les auteurs de dictionnaires pour qui l'unité minimale est le mot sont bien obligés dans la confection même des articles de donner un contexte pour désambiguïser les différentes acceptions. Mais ils le font au coup par coup et sans théorie. Si l'on utilise les classes d'objets pour déterminer un emploi prédicatif, on a l'avantage de pouvoir le décrire en intension et en exten- 
sion, ce qui est un avantage considérable pour le traitement automatique. D’autre part, la linguistique de corpus ne peut pas se passer de la notion d'environnement pertinent, si elle veut trouver dans les textes des informations utiles à la description des mots du lexique, ni de la notion de phrase simple. Enfin, l'entrée d'un article de dictionnaire doit représenter une phrase avec l'indication des restrictions sémantiques qui caractérisent le prédicat et ses arguments, à quoi il faut ajouter les différentes modifications qu'une phrase peut subir dans les textes.

\section{NOTE}

1. Nouveau Petit Robert (1993) Paris: Dictionnaires Le Robert.

\section{RÉFÉRENCES}

Boons, Jean-Paul, Guillet, Alain et LeClère, Christian (1976) : La structure des phrases simples en français. Genève: Droz.

Buvet, Pierre-André (1998) : Détermination et classes d'objets. Langages. 131:91-102.

Fairon, Cédrick (2000): Structures non connexes. Thèse de doctorat. Paris: Université Paris 7.

Gross, Gaston (1996a): Semantische Umgebung der Konnektoren. Leuvense Bijdragen. 84:295311.

Gross, Gaston (1996b): Les expressions figées en français. Paris: Ophrys.

Gross, Gaston (1998): Pour une véritable fonction synonymie dans un traitement de textes. Langages. 131:103-114.

Gross, Maurice (1975): Méthodes en syntaxe. Paris: Hermann.

Gross, Maurice (1981): Les bases empiriques de la notion de prédicat sémantique. Langages. 63:7-52.

Gross, Maurice (1993): Les phrases figées en français. L'Information Grammaticale. 59:36-41. 